\title{
Bubble Impingement and the Mechanisms of Heat Transfer
}

\author{
D.B. Donoghue ${ }^{a}$, A. Albadawi ${ }^{b}$, Y.M.C. Delauréb, A.J. Robinson ${ }^{a}$, D.B. Murray ${ }^{\mathrm{a}}$ \\ ${ }^{a}$ Department of Mechanical $\mathcal{E}$ Manufacturing Engineering, Trinity College Dublin, Ireland \\ ${ }^{b}$ School of Mechanical $\mathcal{E}$ Manufacturing Engineering, Dublin City University, Ireland
}

\begin{abstract}
Heat transfer augmentation resulting from the effects of two phase flow can play a significant role in convective cooling applications. To date, the interaction between a rising gas bubble impinging on a heated horizontal surface has received limited attention. Available research has focused on bubble dynamics and the associated heat transfer has not been reported. To address this, this study investigates the effect of a single bubble impinging on a heated horizontal surface. Local and time resolved heat transfer measurements have been performed for a died orifice to surface distance, with a bubble injection orifice of $1 \mathrm{~mm}$ in diameter. Synchronized high-speed photography and infrared thermography have been utilized to investigate the path of the bubble and the associated heat transfer.
\end{abstract}

Keywords:

Rising bubble, Bouncing bubble, Local heat flow

\section{Introduction}

Fluid agitation and mixing ensuing from two phase flow has an important role to play in convective cooling applications. In some heat exchangers with two phase flow, vapour bubbles may sometimes impinge against the heat exchanger surface and this behaviour can directly affect convective heat flow within the heat exchanger (Atmane \& Murray [1], Cornwell [2]). Investigations into this complex fluid structure interaction have only begun in recent years, most recently by Sato et al. [3]. Bubbly flows are not only confined to heat exchangers, with bubbles being used for a variety of industrial applications such as froth flotation water treatment processes and the production of aluminium foam for example.

Several studies have reported experimental results concerning impingement and subsequent bouncing of a single gas/vapour or liquid bubble on a wall in various fluids [3-14]. Many of these authors investigated bubble impact against deformable surfaces, such as the interface between water and air, where coalescence would most likely occur [3, 4, 6-8, 10], while the remaining authors have investigated the effect of a bubble bouncing against solid surfaces [5, 9, 12-14]. Tsao \& Kock [5] observed the interaction between high

Email address: donoghdb@tcd.ie (D.B. Donoghue)
Reynolds number, moderate Weber number bubbles impacting solid walls. A glass plate was placed at the top of a glass column filled with water. An air bubble with a radius of about $0.5-1 \mathrm{~mm}$ was released from a needle and rose to the surface under the action of buoyancy forces. Their analysis indicated that $95 \%$ of the kinetic energy contained within the bubble was converted to heat via viscous dissipation within the separation film and a part was transformed into surface deformation during the bubble collision with the solid surface.

Surface properties have a significant influence on bubble dynamics; hydrophilic and hydrophobic surface characteristics have been found to influence bubble motion. Krasowska \& Malysa [11] studied the influence of surface roughness of hydrophilic and hydrophobic plates on the kinetics of the bubble attachment. They found that the roughness of the hydrophobic surface is of importance for the kinetics of the bubble attachment. Similarly the degree of roughness directly affects the detachment time of the bubble.

Legendre et al. [9] studied 2.1 to $3.3 \mathrm{~mm}$ liquid Toluene droplets rising in water and bouncing under a horizontal plate. Before the impact with the wall, the drops are spherical and move along a rectilinear trajectory with a constant terminal velocity corresponding to the velocity of a solid sphere of the same diameter and density. During the impact with the wall the drops are deformed and exhibit a shape which is close to ellipsoid.

June 27, 2013 


\begin{tabular}{|c|c|c|c|}
\hline \multicolumn{4}{|c|}{ Nomenclature } \\
\hline$C_{p}$ & specific heat capacity $(\mathrm{J} / \mathrm{kgK})$ & $\chi$ & aspect ratio (Eq. 5) \\
\hline $\operatorname{Re}$ & Reynolds number (Eq. 4) & $\delta$ & surface thickness (m) \\
\hline$S$ & coordinate $(\mathrm{m})$ & $\kappa$ & conductivity (W/mK) \\
\hline$T$ & temperature $(\mathrm{k})$ & $\mu$ & viscosity (Pa.s) \\
\hline$T^{*}$ & dimensionless temperature (Eq. 6) & $\rho$ & density $\left(\mathrm{kg} / \mathrm{m}^{3}\right)$ \\
\hline$U_{T}$ & terminal velocity $(\mathrm{m} / \mathrm{s})$ & $\tau$ & time (s) \\
\hline$V$ & velocity (m/s) & $\theta$ & major axis orientation $\left({ }^{\circ}\right)$ \\
\hline$a$ & minor axis $(\mathrm{m})$ & \multicolumn{2}{|c|}{ Subscripts } \\
\hline$b$ & major axis (m) & $\infty$ & bulk fluid conditions \\
\hline$d_{e}$ & equivalent diameter (m) & $f$ & foil \\
\hline & heat flux $\left(\mathrm{W} / \mathrm{m}^{2}\right)$ & $p$ & paint \\
\hline \multicolumn{2}{|c|}{ Greek Symbols } & $s$ & test surface \\
\hline$\alpha$ & thermal diffusivity $\left(\mathrm{m}^{2} / \mathrm{s}\right)$ & $x, y, z$ & coordinate direction \\
\hline
\end{tabular}

The bouncing was found to be very dissipative, more than $80 \%$ of the energy being lost during the interaction with the wall. For bubble bouncing, the mechanisms responsible for the loss of kinetic energy, as reported by Legendre et al. [9], are located in the surrounding fluid i.e. film drainage and fluid motion parallel to the solid wall. Fujasová-Zedníková et al. [13] studied the interaction of a small bubble with an immersed horizontal solid surface. The effect of a surfactant and surface properties were investigated. They found that the presence of $\alpha$-terpineol in water decreased the bubble velocity and it was found to suppress the bubble's rebound from the surface.

The phenomenon of heat transfer enhancement resulting from bubble motion near a wall is well established, with numerous studies being performed over the years [15-24]. Donnelly et al. [21] investigated the effect of a single rising bubble near a heated vertical wall. The study highlighted two important parameters which were found to significantly enhance heat transfer from the surface: path orientation and bluff body interactions due to the proximity from the surface, which is consistent with other studies $[18,20,22,23]$. Path orientation is linked to the periodic release of vortices $[25,26]$ and these vortices play a key role in convective heat transfer. These results helped explain an early study by Cornwell [2], who investigated the role of bubbly flow in boiling within a tube in a bundle. A significant increase in the heat transfer was observed for tubes exposed to bubbly flow. A particularly significant finding was that the bubbles rising from a lower tube could completely suppress nucleation of new bubbles on an upper tube signifying improved heat transfer. Later Hous- ton \& Cornwell [27], employed the same experimental technique as Cornwell [2] but instead of vapour bubbles, air bubbles were introduced into the system. They confirmed that large scale mixing caused by bubbly-flow is a prominent mechanism of the heat transfer.

While sliding bubble heat transfer enhancement has received some attention in the open literature, impinging and bouncing against horizontal surfaces has not. Yan et al. [28] investigated bubbles impacting under a horizontal curved surface, which is in effect bouncing on a curved surface. They found that the bubbles tended to stick to the surface and cause a hot spot through local dry-out, inducing elevated temperatures, before growing and sliding away from the bottom of the cylinder. This was the first reputed study into the effect of a bubble impacting a surface. A similar study was performed by Atmane \& Murray [1] who measured local heat flux variation due to vapour or air injected bubble motion.

The objective of this study is to experimentally investigate the coupled convective heat transfer and bubble motion due to the effect of a single air bubble rising through water and impacting and bouncing on a heated horizontal surface. Specifically, the time varying convective heat flux distribution is quantified for a single bubble impact and bouncing event and the results explained in terms of the bubble dynamics.

\section{Experimental Apparatus}

The experimental apparatus is shown in Fig. 1. It consists of a tank of $110 \times 95 \times 195 \mathrm{~mm}^{3}$ constructed from 3 $\mathrm{mm}$ thick glass with a horizontally mounted test surface 
placed at the top. The tank contains an adjustable bubble injection orifice, a controllable heated surface and a bulk water thermocouple. A high-speed infrared camera and two CCD cameras are mounted directly above and horizontally at the sides of the test section respectively. To capture rapid movement of the bubble as it rises, high intensity lighting is required to enhance the outline of the bubble. This is provided by three high intensity light emitting diode (LED) strips mounted behind each camera. A diffusive screen is placed between the lights and glass tank to create a uniform light sheet. Two NAC Hi-Dcam II digital high-speed colour cameras are used in these experiments. Both high speed cameras give a spatial resolution of approximately 37 $\mu \mathrm{m} /$ pixel.

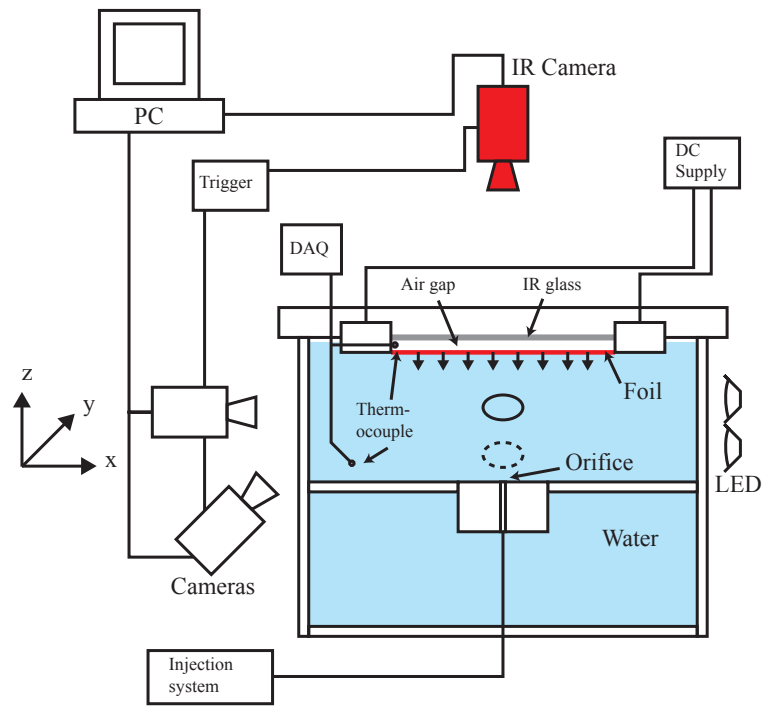

Figure 1: Experimental set-up. The High-speed cameras are orientated perpendicular to one another.

A FLIR SC6000 high resolution, high speed infrared camera is used to capture spatial and temporal changes in the surface temperature during bubble impingement events. The camera has a $640 \times 512$ pixel focal plane array and an InSb 3-5 $\mu m$ sensor which is vacuum sealed within the cooler assembly. For the present study, the field measured by the camera is an area measuring $34.6 \times 34.6 \mathrm{~mm}^{2}$, corresponding to a spatial resolution of $216 \mu \mathrm{m} /$ pixel. All three cameras are set to record at a frequency of $1000 \mathrm{~Hz}$. This is achieved by means of a Thurlby Thandar TG300 series function generator which produces a square wave signal at a frequency of $1000 \mathrm{~Hz}$, which is fed directly to the IR camera. The IR camera is, in turn, connected to the PCI controller card
Table 1: Foil and paint properties.

\begin{tabular}{|l|l|}
\hline Density $\rho_{f}$ & $8900 \mathrm{~kg} / \mathrm{m}^{3}$ \\
Thermal conductivity $k_{f}$ & $19.5 \mathrm{~W} / \mathrm{mK}$ \\
Specific heat $C_{f}$ & $390 \mathrm{~J} / \mathrm{kgK}$ \\
Thermal diffusivity $\alpha_{p}$ & $1.7 \times 10^{-7} \mathrm{~m}^{2} / \mathrm{s}[29,30]$ \\
\hline
\end{tabular}

for the high speed cameras. The IR camera has an exposure time of $0.85 \mathrm{~ms}$, while both high speed cameras have an exposure time of $0.5 \mathrm{~ms}$.

The bubble injection system is mounted on a movable platform, which allows the injection point to be adjusted to varying distances from the test surface. For the present study the distance was $30 \mathrm{~mm}$. The injection orifice is a $1 \mathrm{~mm}$ hole machined into an aluminium base which is connected to a Hamilton GASTIGHT 10022.5 $\mathrm{ml}$ syringe by means of a $300 \mathrm{~mm}$ length of tube with a $0.5 \mathrm{~mm}$ internal diameter. An accurate bubble injection rate is achieved by using a KD Scientific model 220 series infusion pump set to an injection flow-rate of 100 $\mathrm{ml} / \mathrm{hr}$ for the present study.

The tank is filled with ultra-pure water, purified with a Millipore purification system. The water is maintained at a temperature of $22 \pm 0.5^{\circ} \mathrm{C}$.

The test surface consists of a metal foil, the lower side of which is in contact with the water while the upper side is insulated by means of infrared transparent glass. The test surface is a $10 \mu \mathrm{m}$ thick Constantan Alloy Cu55/Ni45 rolled foil supplied by Goodfellow Ltd. The foil is clamped between two busbars using silver based electrically conductive epoxy. The top side of the foil viewed by the IR camera is coated with matt black paint with an emissivity of 0.95 . The thickness was determined to be $10.3 \pm 0.1 \mu \mathrm{m}$. The foil and paint properties are shown in Table 1.

The foil is insulated on the upper surface by a $3 \mathrm{~mm}$ air gap. The air gap is maintained by a Calcium Fluoride (CaF2), $1 \mathrm{~mm}$ thick IR transparent viewing window. This polished glass window has a very high percentage transmissibility, approximately $95 \%$ for a depth of $1 \mathrm{~mm}$.

In order to evaluate the convective heat flux, a successive local energy balance is applied to the heater. Eq. 1 accounts for generated heat flux ( $q_{\text {gen }}^{\prime \prime}$, Eq. 2$)$, conduction through the $3 \mathrm{~mm}$ air gap to the IR glass $\left(q_{\text {cond }}^{\prime \prime}\right)$ and radiation from the surface $\left(q_{\text {rad,total }}^{\prime \prime}\right)$. Lateral heat conduction and heat storage within the foil are also accounted for in Eq. 1 which is solved by applying a second order central-difference approximation to the spatial derivatives and a first order central-difference approximation to the time derivative $[29,31,32]$. Results 


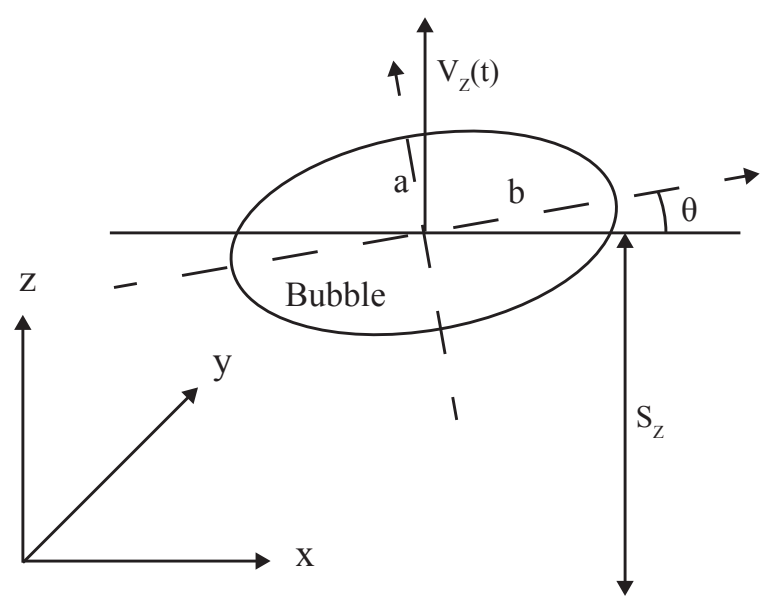

Figure 2: Schematic of the bubble, with a velocity of $V_{Z}$ in the vertical direction, with $S_{Z}$ being the height of the bubble's centroid above the orifice. The variable $\theta$ is the orientation of the major axis $b$ with respect to the horizontal.

in this investigation are discussed in terms of the parameters: Terminal velocity $U_{T}$, Reynolds number $R e$, dimensionless surface temperature $T^{*}$ and bubble aspect ratio $\chi$, where $a$ and $b$ are the minor and major axis lengths as depicted in Fig. 2. The above parameters are defined as follows,

$$
\begin{gathered}
q_{\text {conv }}^{\prime \prime}=q_{\text {gen }}^{\prime \prime}-q_{\text {cond }}^{\prime \prime}-q_{\text {rad,total }}^{\prime \prime}+ \\
\left(k_{f} \delta_{f}+k_{p} \delta_{p}\right)\left(\frac{\partial^{2} T}{\partial x^{2}}+\frac{\partial^{2} T}{\partial y^{2}}\right)- \\
\left(\rho_{f} C p_{f} \delta_{f}+\rho_{p} C p_{p} \delta_{p}\right) \frac{\partial T}{\partial t} \\
q_{g e n}^{\prime \prime}=\frac{V I}{A_{\text {surf }}} \\
U_{T}=\sqrt{V_{x}^{2}+V_{y}^{2}+V_{z}^{2}} \\
R e=\frac{\rho U_{T} d_{e}}{\mu} \\
\chi=\frac{a}{b} \\
T^{*}=\frac{T_{s, i j_{\tau}=n}-T_{\infty}}{\bar{T}_{s, \tau=0}-T_{\infty}}
\end{gathered}
$$

where $T_{s, i j_{\tau=n}}$ is the local surface temperature at a particular time $n, \bar{T}_{s, \tau=0}$ is the averaged surface temperature at the beginning of a test and $T_{\infty}$ is the bulk water temperature.

\subsection{Uncertainty Analysis}

The uncertainty in the convective heat flux due to the motion of the bubble and pursuing wake is evaluated utilizing an error propagation analysis on Eq. 1 as described by Coleman [33]. The uncertainty for each term in Eq. 1 has been evaluated in accordance with Kim et al. [34] at a confidence level of 95\%. For both the lateral conduction and heat storage terms an additional term is added to the standard propagation equation for a multi-variable function, which is known as the covariance term. In order to determine how correlated a pixel is to its neighbouring pixel and similarly to same pixel but with a time shift, both a cross-correlation and autocorrelation analysis was performed and used to determine the degree of correlation. The resulting uncertainty in the measured heat flux ranges from 12-21\%, with the maximum being at the highest convective heat flux level due to the additional error associated with the lateral conduction and thermal storage.

\section{Results and Discussion}

\subsection{Bubble Dynamics}

Once a bubble detaches from the orifice it begins to accelerate and rise through the bulk liquid. Fig. 3 illustrates a bubble detaching from a $1 \mathrm{~mm}$ orifice before rising $30 \mathrm{~mm}$ to the horizontal surface. In this case it takes the bubble approximately $100 \mathrm{~ms}$ to rise $30 \mathrm{~mm}$ and impact the surface. The bubble's centre of area is determined to be the centroid of a 2D area, while assuming a constant air density. The developing rise velocity and rise location versus time for this bubble sequence are shown in Fig. 4 (a). The equivalent bubble diameter for the current case is $d_{e}=3.37 \mathrm{~mm}$, which was determined by equating the volume of the bubble to that of a sphere. The maximum terminal velocity attained by the bubble is $U_{T}=333 \mathrm{~mm} / \mathrm{s}$, with a corresponding Reynolds number of $R e=1453$.

What is clear from Fig. 4 (a), is the large variation in the rise velocity following the bubble's departure. Once the bubble departs the orifice, it follows a straight path for approximately $14 \mathrm{~mm}$. This occurs at a time of around $60 \mathrm{~ms}$ in Fig. 3 and 4 (b) where the major axis orientation begins to change. Beyond this time the bubble begins to deviate from its straight vertical path in both the $x$ and $y$ directions. This directional change is evident in the way the major axis of the bubble tilts by a maximum of $10^{\circ}$ (illustrated in Fig. 4 (b) at a time of 90 $\mathrm{ms}$ ); the direction of tilting was found to be random and 


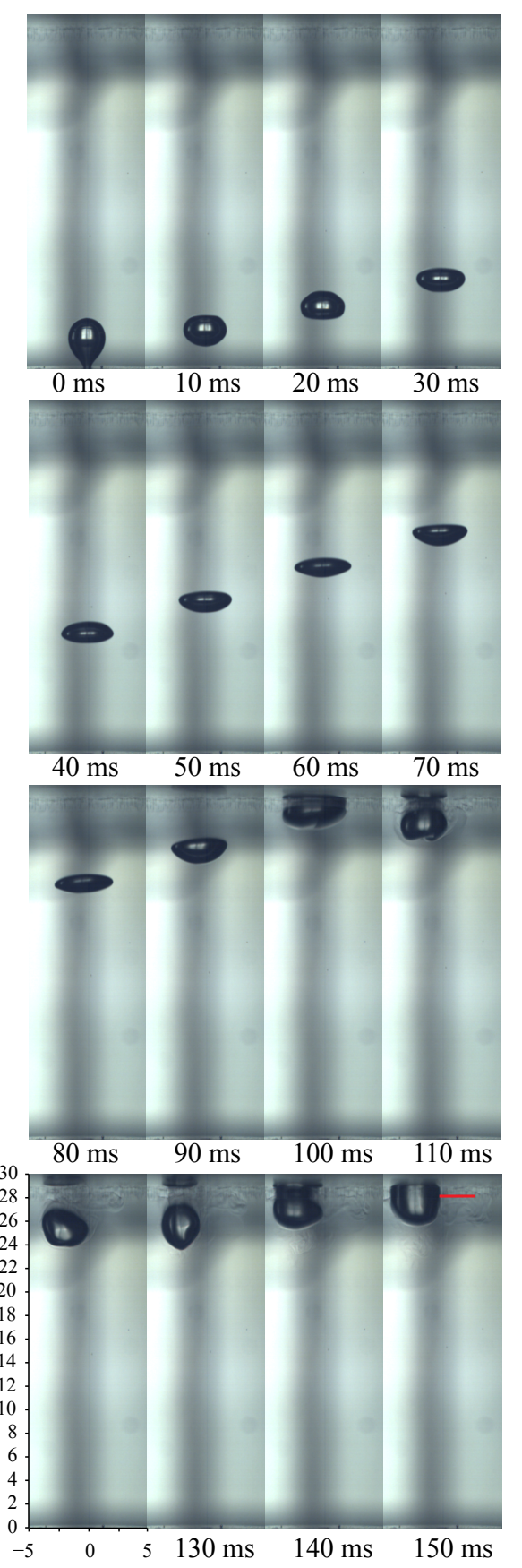

Figure 3: Sequence depicting the rise of a single bubble from a $1 \mathrm{~mm}$ orifice. All images are in $\mathrm{mm}$, with the scale shown on the bottom left image. The red horizontal line shown at a time of $150 \mathrm{~ms}$, indicates the approximate location of the contact line.

the bubble retains this tilt until impact. The bubble's vertical rise location $S_{Z}$, is shown in Fig. 4 (a) after a time of $70 \mathrm{~ms}$.
As the bubble rises, the velocity fluctuations (between times $20-100 \mathrm{~ms}$ ) are linked to the deformation of the bubble. This is illustrated in Fig. 4 (b), where the aspect ratio varies from 0.35 to 0.95 in this period. These fluctuations indicate that the bubble is becoming flatter as it rises, which is also clear from the sequence in Fig. 3. The maximum rise velocity was found to occur just prior to impact at $95 \mathrm{~ms}$.

Once the bubble impacts the surface (point I in Fig. 4 (a)), the bubble shape flattens considerably, with an aspect ratio as low as $\chi \approx 0.2$. The surface tension energy stored in the flattened bubble is partially released as the bubble springs back from the surface. During the rebound event the bubble aspect ratio increases to the region of $\chi \approx 0.9$, signifying a much more spherical bubble as depicted in Fig 3 for $t=110 \mathrm{~ms}$. The bubble's shape was found to oscillate quite significantly during its rebound from the surface.

When the bubble impacts the surface, a portion of its kinetic energy is converted into surface energy, while some is dispersed through viscous dissipation $[5,9,12]$. During the post impact recoil event, when the bubble is becoming more spherical and moving downward, the stored surface energy from the impact is converted to kinetic and potential energy as the bubble accelerates away from the surface. This recoil event is signified by an aspect ratio tending towards unity (Fig. 4 (a)) and a negative velocity (Fig. 4 (b)). The initial acceleration phase is followed by a deceleration phase to the point at which $V_{Z}=0 \mathrm{~mm} / \mathrm{s}$ when $S_{Z}$ reaches a local minimum i.e. the bubble reaches its furthest distance from the surface during the bouncing phase. This occurs at $t \approx 130$ $m s$ and at this moment the bubble is quite spherical as shown in the photograph in Fig. 3 as well as $\chi \approx 1$ in Fig. 4 (a). At this moment the bubble begins moving upward and accelerates towards the surface. This time, however, the rise velocity at the second impact $\left(V_{Z} \approx\right.$ $150 \mathrm{~mm} / \mathrm{s})$ is much lower than the first impact $\left(V_{Z} \approx 350\right.$ $\mathrm{mm} / \mathrm{s})$ as the bubble had less distance to travel. In fact, when the bubble impacts the surface this second time, a triple contact line is immediately formed and the bubble is attached to the surface with no further rebound (point III in Fig. 4 (a)).

Subsequent to this the contact line spreads forming an increasingly large dry area between the bubble and the foil. During this spreading phase the bubble aspect ratio decreases due to the growing contact perimeter which causes $V_{Z}$ to be positive, though decreasing towards zero (Fig. 4 (a)). At this point the bubble motion has stopped, barring small motions due to the impact of the ensuing wake. 


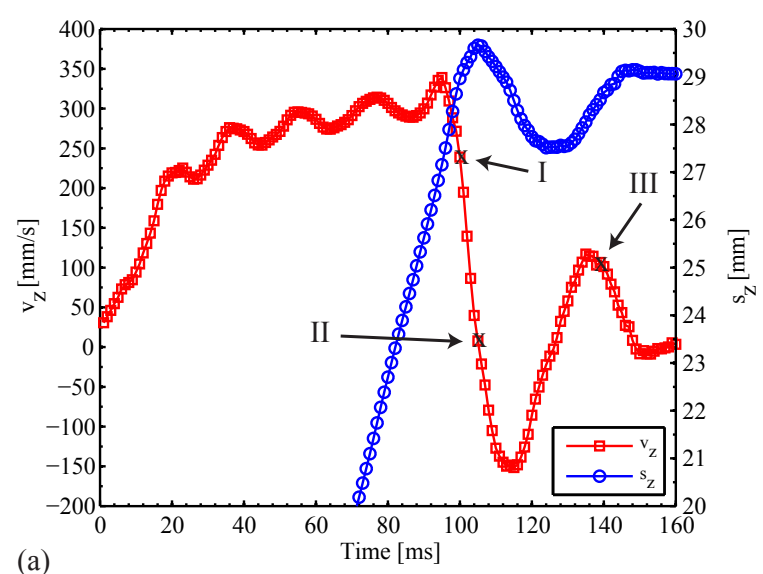

(a)

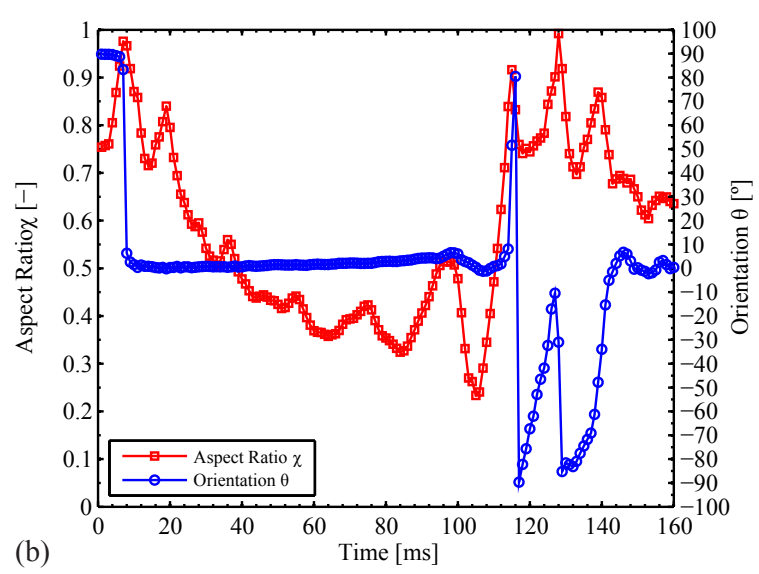

Figure 4: (a) Centroidal rise velocity and rise location versus time corresponding to the image sequence depicted in Fig. 2. Only the rise height above $20 \mathrm{~mm}$ is shown as this is the region of most interest and (b) eccentricity and major axis orientation. $I$ is the moment of impact, $I I$ is the moment when the bubble leaves the surface and III is the time at which a triple contact line forms signifying bubble attachment to the surface.

\subsection{Bubble Rise, Impact and Bouncing Phases}

In the present study the baseline convective heat flux i.e. for natural convection alone, was $q^{\prime \prime}=7300 \mathrm{~W} / \mathrm{m}^{2}$ which resulted in a uniform and steady wall temperature of approximately $51^{\circ} \mathrm{C}$, for the duration of the experiment. By definition, the non-dimensional temperature distribution is initially equal to unity across the entire foil.

Figure 5 plots the maximum and minimum dimensionless wall temperature histories from the time at which the bubble was released from the orifice to the time at which the bubble is at rest on the surface. Increases in $T^{*}$ are indicative of decreased heat transfer whereas decreasing $T^{*}$ is indicative of improved heat transfer. From Fig. 5, four regions have been identified with regard to the coupling of the fluid mechanics and the heat transfer.

The first regime corresponds to heat transfer due to natural convection to water alone (I). This regime extends from when the bubble departs from the orifice until the bubble impacts the heated surface. As mentioned, $T^{*}$ is unity throughout the foil so that $T_{\text {max }}^{*}=T_{\text {min }}^{*}=1$ during the bubble release and rise phase. The second regime extends from when the bubble has initially impacted the surface until it becomes attached to the surface (II). The third is relevant only for the maximum temperature on the surface of the foil (III); it commences when the bubble becomes attached and continues until the slope of the temperature rise changes sharply. The final regime also starts at the same time as regime (III) and extends from when the bubble becomes attached to the surface until the point at which the foil has again reached steady state i.e. natural convection (IV). The present paper will focus on the effect of the bouncing bubble i.e. regime II.

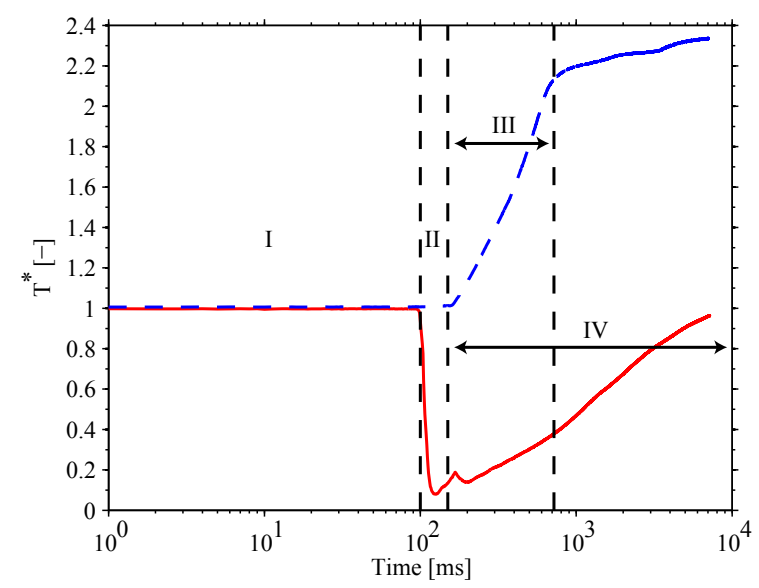

Figure 5: Minimum surface temperature (solid - red) and maximum surface temperature (dashed - blue). (I) is the natural convection regime, (II) bouncing bubble dynamics, (III) maximum temperature beneath the attached bubble and (II-IV) the regime of shed vortices interaction with the surface heated surface.

Regime (II), marked in Fig. 5, is associated with the movement of the bubble and its interaction with the surface. Once the bubble is within the thermal boundary layer region, the influence of the bubble is noticeable on the surface. This is mainly because the bubble acts as a bluff body, pushing fluid ahead of it, during its rise. This effect was found to occur up to $4 \mathrm{~ms}(\approx 1 \mathrm{~mm}$ away from the surface) prior to direct impact on the surface. Once the bubble impacts the surface, bubble surface shape deformation takes place as previously discussed. Fig. 6 depicts the change in dimensionless surface temperature, 


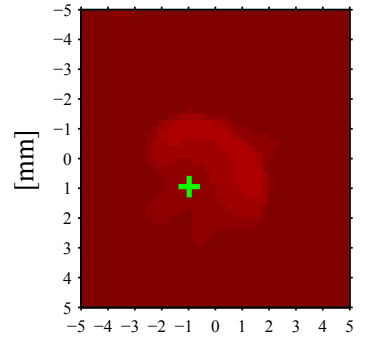

$100 \mathrm{~ms}$

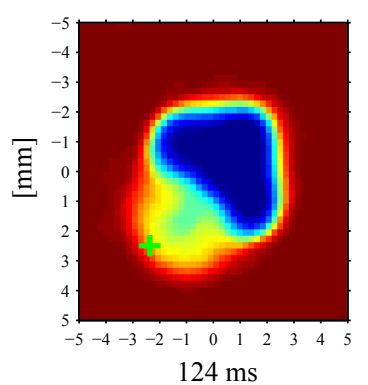

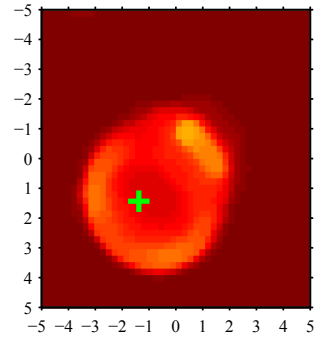

$106 \mathrm{~ms}$

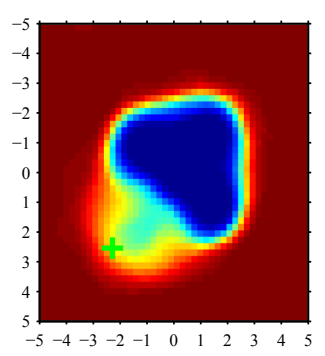

$130 \mathrm{~ms}$

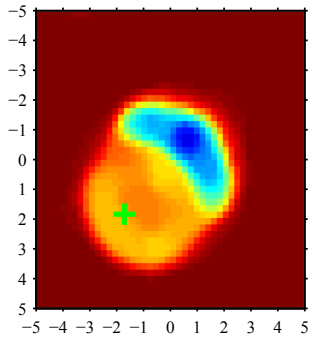

$112 \mathrm{~ms}$

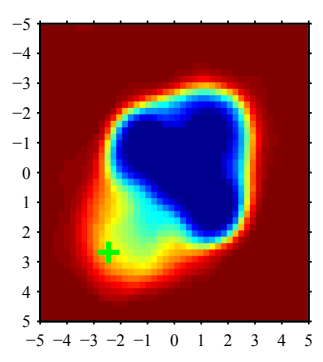

$136 \mathrm{~ms}$

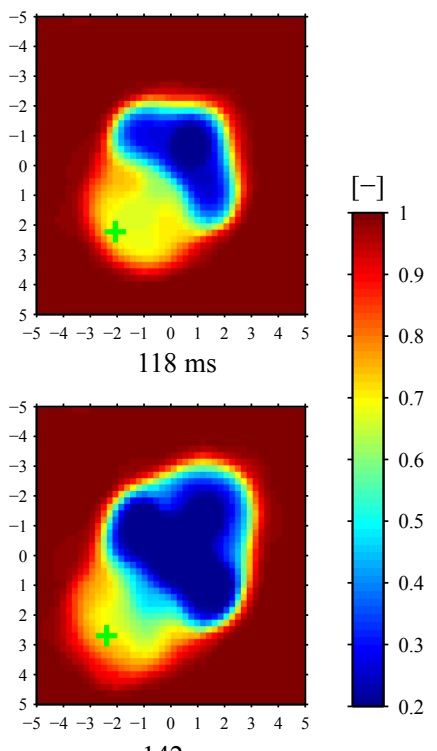

$142 \mathrm{~ms}$

Figure 6: Sequence depicting the change is dimensionless temperature $\left(T^{*}\right)$ due to the bubble motion. This sequence relates to regime II in Fig. 5. The green cross indicates the approximate location of the bubble's centroid.
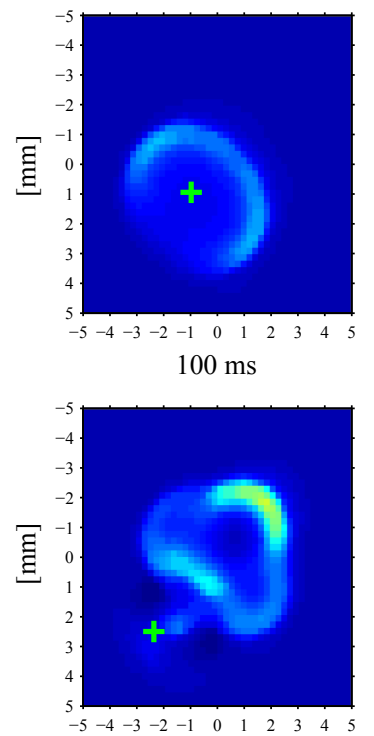

$124 \mathrm{~ms}$

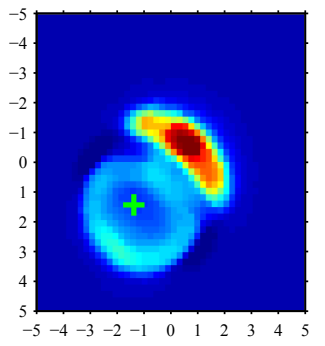

$106 \mathrm{~ms}$

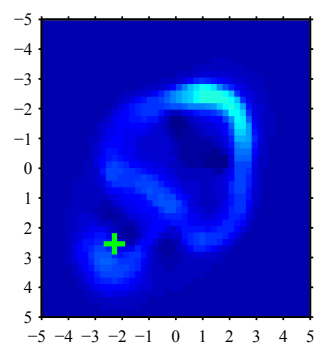

$130 \mathrm{~ms}$

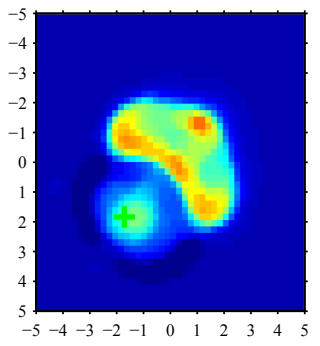

$112 \mathrm{~ms}$

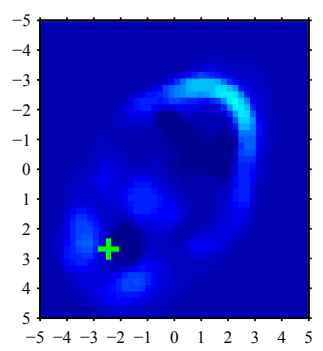

$136 \mathrm{~ms}$

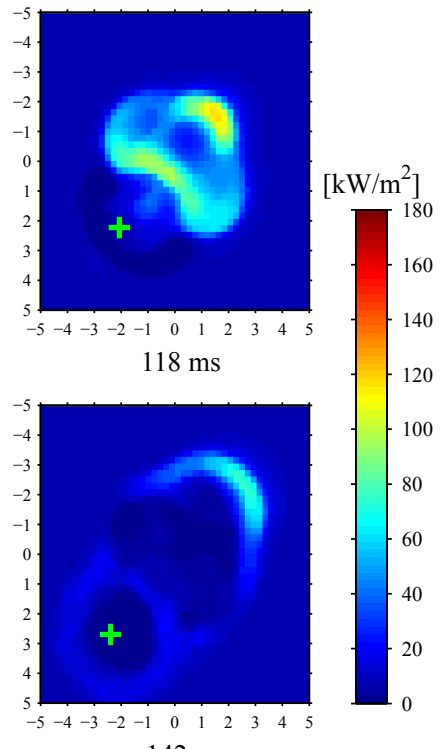

$142 \mathrm{~ms}$

Figure 7: Sequence depicting the change in convective heat flux $\left(q_{\text {conv }}^{\prime \prime}\right)$ to the fluid due to the bubble motion. This sequence relates to regime II in Fig. 5 and the temperature sequence in Fig. 5. The green cross indicates the approximate location of the bubble's centroid. The heat flux scale in this case is restricted to $0-180 \mathrm{~kW} / \mathrm{m}^{2}$ for clarity.

defined by Eq. 6, from when the bubble first impacts the surface until bubble attachment to the surface. It is clear from Fig. 6 that the movement of the bubble (the bub- ble's centroid is highlighted by a green cross) is down and to the left, whereas the region of significant cooling moves in the opposite direction. 
The convective heat flux distributions corresponding to the temperature sequence in Fig. 6 are shown in Fig. 7. What is initially evident is the symmetry of the both plots (Fig. 6 and 7) within the bubble movement and the following wake of the bubble, if viewed at 45 degree angle, beginning at the bubble's centroid.

The direct enhancement as a result of the bubble impacting the surface is quite limited in time, lasts for a mere 4-6 ms (at a time of $106 \mathrm{~ms}$, Fig. 7) before being dominated by the enhancement due to the fluid motion in the vicinity of the bubble. Within the region of the bubble, visible at a time of $100 \mathrm{~ms}$, there is a clear variation in heat flux and temperature. The extremities of the bubble convect more heat when compared to the centre of the bubble; this may be due primarily to cupping/dimpling of the bubble prior to impact. This dimpling phenomenon has been observed by Zapryanov \& Tabakova [35] and Tsao \& Kock [5], and results from a viscous effect, trapping fluid between the deformable bubble and the rigid boundary. As the bubble approaches the surface, the bubble must displace fluid in order to rise; this results in higher pressure at the leading edge of the bubble, which has the effect of pushing fluid to regions of lower pressure.

\subsection{Local Variation in Heat Flux and Temperature}

In order to evaluate the complex process of heat flow in the presence of a bubble impacting the surface, specific locations of interest have been chosen. A time trace of heat flux and temperature at these points will be presented in the following section.

The four points chosen are at the rear, centre, front and edge of the bubble, when viewed at an angle of 45 degrees, at a point in time when the bubble has impacted the surface. These four points are highlighted in Fig. 8 with $a$ (edge), $b$ (centre), $c$ (front) and $d$ (rear), marking their locations. The highlighted times in Fig. 8 and following figures, relate to the time after the bubble has impacted the surface at $100 \mathrm{~ms}$. Each location corresponds to a single point in the temperature map and it can be seen from the sequence of images that the bubble moves with respect to these fixed locations.

The motion of the bubble can be separated into two sections (A and B) as illustrated in Fig. 8. Section A relates to the bubble impact and the beginning of its rebound from the surface, while section $B$ refers to the bouncing of the bubble, which is related to its rebound and secondary approach to the surface. As the bubble impacts the surface (section A) its outline can be inferred from the convective heat flux distribution shown in Fig. 8 at a time of $2 \mathrm{~ms}$. As the flattened bubble begins to recover its shape, the convective heat flux distri-

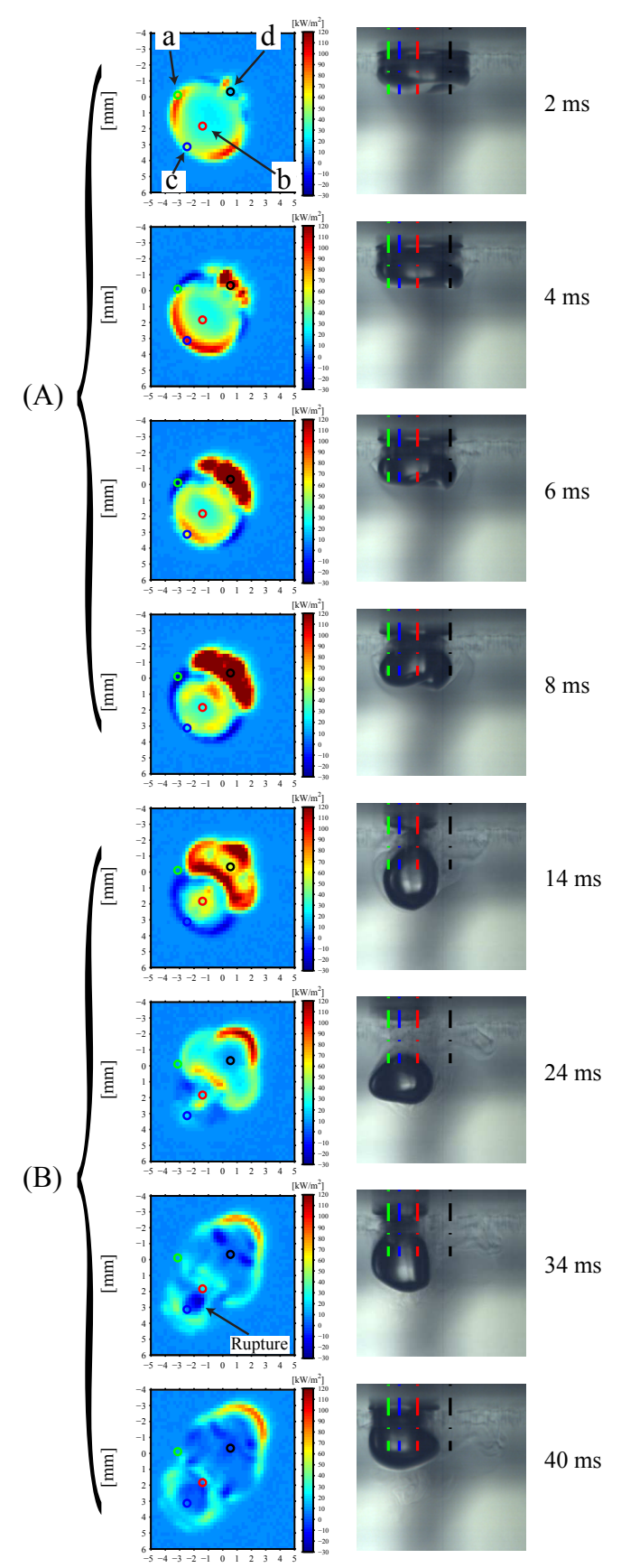

Figure 8: Sequence depicting the impact of the bubble initially shown in Fig. 3. The times shown are related to points of interest in later figures. The left hand side figures are convective heat flux maps while the right hand side is the location of the bubble on the surface. Four points of interest are highlighted with coloured circles (heat flux map), while vertical lines are shown in the right hand images, highlighting the approximate location of the points on the surface.

bution similarly changes shape with regions of low and high heat transfer. At times of $6-8 \mathrm{~ms}$, a large varia- 
tion in convective heat flux is observed at the rear of the bubble (point $d$ ).

During section B the bubble continues its rebound from the surface, bouncing approximately $2.5 \mathrm{~mm}$ away from the surface at a time of $24 \mathrm{~ms}$, before moving upward again. During this time, large variations in convective heat flux occur at the rear of the bubble rather than within the bubble's footprint, where only small variations in convective heat flux occur. At a time of $34 \mathrm{~ms}$ the bubble begins to attach to the surface and a triple contact line is formed which subsequently spreads until the bubble reaches its final equilibrium shape.

\subsubsection{Point a}

Fig. 9 illustrates the first point of interest, which is the point marked $a$ in Fig. 8 at approximately $(-3,0)$ $\mathrm{mm}$. Point $a$ is at the edge of the bubble (at 45 degrees with respect to the $x$ and $y$ axis) during the initial impact, although the bubble's final resting position results in it being a few $\mathrm{mm}$ away from the bubble edge. The dashed black line in Fig. 9 indicates the heat flux associated with the volumetric heat generation which is approximately equal to the convective heat flux over the entire field of view when no bubble is present. The solid red line is the bubble affected convective heat flux, while the dashed green line is the heat flux associated with the change in stored energy within the foil.

During the impact event $(A)$ in Fig. 9 a large spike in convective heat flux is observed at a time of $2 \mathrm{~ms}$ with a value approaching $80 \mathrm{~kW} / \mathrm{m}^{2}$; a corresponding drop in surface temperature is observed. The surface temperature reaches a local minimum of $47.5^{\circ} \mathrm{C}$ at $4 \mathrm{~ms}$, after which the temperature increases again. Since the temperature is decreasing, so too is the energy storage term since it is this energy that allows the convective heat flux to increase significantly above the generated heat flux. At a time of $8 \mathrm{~ms}$ the convective heat flux drops below zero to a value of approximately $-27 \mathrm{~kW} / \mathrm{m}^{2}$. This would indicate that the foil received a rapid input of heat, after being a region of high cooling $6 \mathrm{~ms}$ earlier. The only significant source of warm fluid that could account for the rise in temperature is the thermal boundary layer and this will be discussed later. A secondary source of heat input is the air trapped within the $3 \mathrm{~mm}$ gap above the foil, this will be shown to be significant at a specific time and will be discussed later. At a time of 14 $m s$ the surface temperature has reached a maximum and the convective heat flux has increased to the level of that generated within the foil. During this stage the storage term increases and becomes positive and larger than the generated heat flux which also indicates that additional
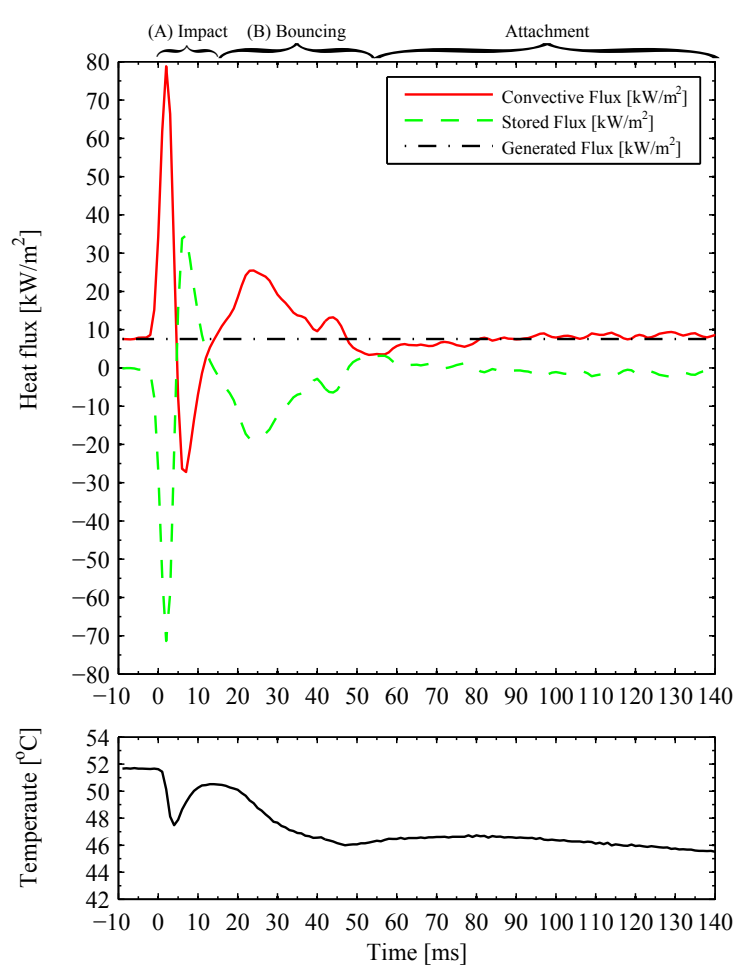

Figure 9: Time trace of heat flux and temperature at Point $a$, shown in Fig. 8. The $x$ axis time begins from when the bubble impacts the surface. The upper graph illustrates the change in surface heat flux, with the lower graph illustrating the change in surface temperature.

(A)

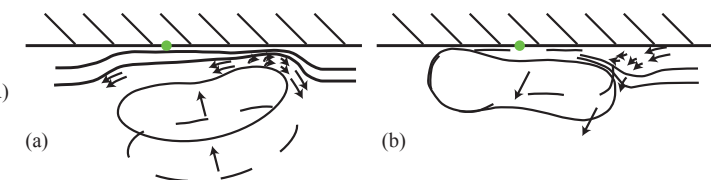

(B)

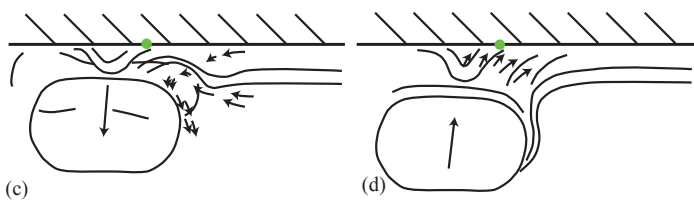

(c)

Figure 10: Sketches showing (a) the bubble approach (-4 - -2 $m s$ prior to impact), (b) bubble initial rebound from the surface $(2-4 m s)$, (c) complete rebound from the surface $(10-18 \mathrm{~ms})$ and (d) fluid flow during bubble's second impact $(28-32 \mathrm{~ms})$. The green circle indicates the approximate location of the current point of interest.

energy is transferred into the foil at this location from the liquid.

Fig. 10 (A) illustrates both bubble and fluid motion during the bubble's impact and initial rebound. During the bubble approach a pressure gradient is produced. 
This pressure gradient begins to move fluid within the thermal boundary layer. Fig. 10 (a) illustrates the approaching bubble, which is believed to push cold fluid towards the warm surface, while the warm boundary layer makes way. Initially the thermal boundary layer was estimated to have an approximate thickness of 2 $\mathrm{mm}$, while during the initial impact this could be reduced to $4-8 \mu \mathrm{m}[36,37]$. The bubble is not directly in contact with the surface, this film layer separates them and the boundary layer thinning is what causes the large increase in the convective heat transfer with the associated drop in temperature. At the time of $4 \mathrm{~ms}$, the first edge of the bubble to impact the surface begins to rebound (Fig. 10 (b)). As the bubble pulls away from the surface, due to mass conservation, fluid must replace the space which was previously occupied by the bubble. As the bubble rebounds the gap between the surface and the bubble remains quite small, thereby only allowing the developed thermal boundary layer access to the previously cooled surface as depicted by Fig. 10 (b). The hot fluid, being warmer than the cooled surface, causes the negative convective heat flux observed in Fig. 9.

During the initial phase of the bouncing event (B) in Fig. 9 the convective heat flux once again increases to above that of the generated heat flux. The enhanced convective cooling draws on energy stored within the foil causing the temperature to decrease with an associated reduction in the stored energy. At a time of $24 \mathrm{~ms}$ the convective heat flux reaches a local maximum after which it reduces, seemingly tending towards the generated heat flux. However, a small inflection is observed at $\mathrm{t}=34 \mathrm{~ms}$ followed by a small secondary peak; this precedes an asymptotic decrease to approximately that of the generated heat flux. During this final phase the storage term decreases to zero as the temperature profile plateaus.

Fig. 10 (B) illustrates the salient features of the bubble bouncing event and coincides with the images in Fig. 8 (B). Initially, between $14 m s \leq \mathrm{t} \leq 24 m s$, the bubble is moving downward away from the surface (Fig. 10 (B) (a)) with a maximum velocity of $150 \mathrm{~mm} / \mathrm{s}$. As the bubble moves cold fluid rushes in to replace the volume occupied by the bubble and this fluid motion is what causes the increasing and positive convective heat flux observed in Fig. 9 during this time period. At $\mathrm{t} \approx 24$ $m s$ the bubble reaches its maximum bounce distance away from the surface; this roughly corresponds with the local maximum in the convective heat flux. Since the fluid motion and associated mixing disrupt the thermal boundary layer and the fluid motions subsequently dissipate, there is a phase of decreasing convective heat flux. This is likely due to the re-growth of the ther- mal boundary layer whereby its thickness increases with time. This, in turn, increases its effective thermal resistance.

At $\mathrm{t} \approx 34 \mathrm{~ms}$ a small inflection in the convective heat flux profile is observed. From close inspection of the video and thermal images this inflection is caused by the rupture of the bubble forming the triple contact line with the surface. This is identified in Fig. 8 and is characterized by a region of low convective heat flux where there exists a dry patch beneath the foil. Once the triple contact line is formed it begins to spread radially outward causing a ring-shaped region of enhanced convection around the rim of the bubble with a circular region of low convective heat flux within the interior. This is clearly visible in the last image in Fig. 8 and is the mechanism responsible for the secondary peak in the convective heat flux which occurs at $\mathrm{t} \approx 44 \mathrm{~ms}$ since Point $(a)$ is located outside of the bubble after it reaches its final resting position. In this regard, since Point $(a)$ exists outside of the bubble perimeter the convective heat flux asymptotically approaches the generated heat flux as the fluid motions dissipate with some small undulations. These are likely to be due to fluid motions induced by the bubble as surface tension forces draw its shape from a distorted $3 \mathrm{D}$ shape to a spherical $2 \mathrm{D}$ equilibrium shape.

\subsubsection{Point b}

As depicted in Fig. 8, Point $(b)$ is located roughly above the centre of the bubble when it first impacts the surface. Compared with Point $(a)$ which is located at the edge of the bubble, it is clear from the sequence of images in Fig. 8 that the convective heat transfer characteristics of Point $(b)$ are notably different.

In a similar manner to Fig. 9, Fig. 11 shows the temporal variation of the generated, convective and storage heat fluxes along with the surface temperature associated with Point $(b)$. Fig. 12 shows interpretive sketches of the impact event (A) and the subsequent bounce event (B) with the relative position of Point (b) indicated.

Prior to impact $(\mathrm{t}<0)$ the convective heat flux rises slowly; it subsequently increases at a notably higher rate at the time of impact. Even still, compared with that of Point $(a)$ at the edge of the bubble, the rate of increase in the convective heat flux is lower for Point $(b)$ at the time of impact up to an initial primary local maximum at approximately $\mathrm{t} \approx 4 \mathrm{~ms}$, the time associated with the first impacting side of the bubble lifting from the surface. After this the convective heat flux increases sharply, reaching a peak heat flux of $90 \mathrm{~kW} / \mathrm{m}^{2}$ at $\mathrm{t}=13$ $m s$, which is the time at which the bubble begins to rebound from the surface. However, it is different to Point 

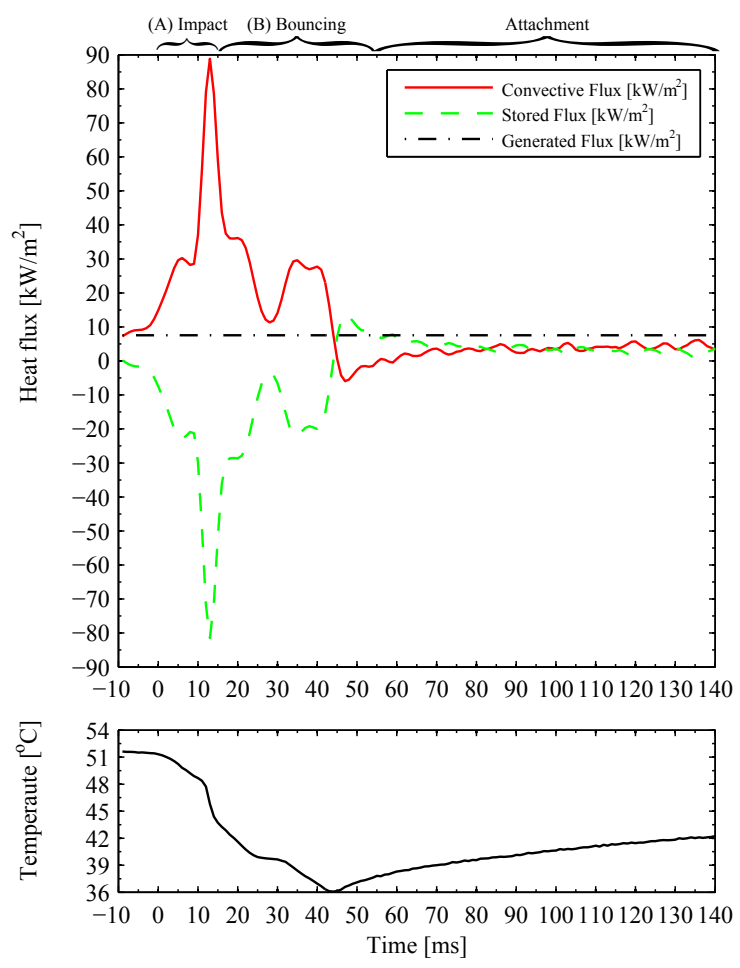

Figure 11: Time trace of heat flux and temperature at Point $b$, shown in Fig. 8. The $x$ axis time begins from when the bubble impacts the surface. The upper graph illustrates the change in surface heat flux, with the lower graph illustrating the change in surface temperature.

(A)

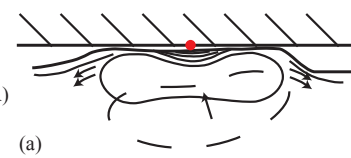

(a)

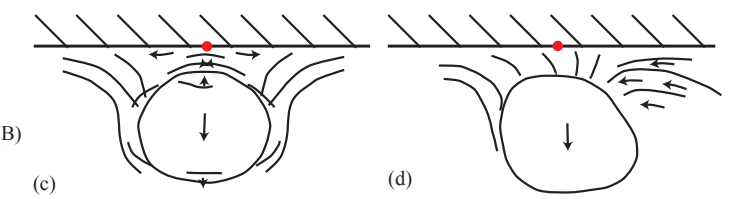

Figure 12: (a) Schematic of fluid flow during impact (-2 to $2 \mathrm{~ms}$ ), (b) during retraction from the surface $(\tilde{1} 0 \mathrm{~ms})$, (c) dimple inversion $(11-$ $13 \mathrm{~ms}$ ) and (d) side flow of fluid due to vortices $(18-20 \mathrm{~ms})$. The red circle indicates the approximate location of the current point of interest.

(a) in the sense that the peak heat flux at the centre of the bubble is $10 \%$ higher and occurs at the end of the impact event, as opposed to at the beginning. This will be discussed in the next section.

Fig. 12 (a) shows a sketch of the bubble shape as de- rived from close inspection of the high speed videos. As shown, when the bubble impacts the surface cupping occurs which traps liquid between the bubble and the heated surface. The thermal boundary layer during the bubble's approach is quite thick $(2 \mathrm{~mm})$, while the approximate depth of the cupping, measured prior to impact, is $>0.5 \mathrm{~mm}$. The fluid contained within the cupped bubble is now comprised of both hot and cold fluid collected during the cupping process and this acts to insulate the surface from direct contact with the bubble. As compared to Point $(a)$, there is less thinning of the boundary layer and as a result the initial peak in the convective heat transfer is less for Point $(b)$. However, as the bubble bounces and rebounds from the surface, as depicted in Figs. 12 (b) and (c), it seeks to recover its spherical shape from the initial disk-like shape upon impact. This causes the dimple to invert as shown in Fig. 12 (c). This inversion process pushes the cold fluid contained within the dimple towards the surface and may explain the sharp increase in heat transfer. Similar to Point $(a)$, the increased convective heat flux draws more energy than is generated in the foil. This causes decrease in the storage heat flux and an associated decrease in the local temperature.

Recall for Point $(a)$ at the edge of the bubble, the wall temperature was found to increase shortly after the initial spike in heat flux due to heat being added to the foil by generation and convection. This is not the case at the centre of the bubble where the convective heat flux remains positive and above the generated heat flux so that the temperature at Point $(b)$ continually decreases. This occurs at the beginning of the bouncing event (14 $m s \leq \mathrm{t} \leq 24 \mathrm{~ms}$ ) as the bubble is moving away from the surface and approaches a local minimum in the convective heat flux at $\mathrm{t} \approx 24 \mathrm{~ms}$, the time when the bubble is furthest from the surface. The small inflection in the convective heat flux is believed to be due to the interaction between the bouncing bubble, moving away from the surface, and the wake that was generated behind the bubble during the initial bubble approach to the surface. Fig. 12 (d) illustrates the movement of fluid on the right hand side of the bubble at a time of between 18 to 28 $m s$. This moving fluid originated from the wake of the impacting bubble and mixes with the wake formed by the bubble as it rebounds from the surface and contains a mixture of cool and warm fluid. This enhanced mixing causes the rate at which the convective heat flux decreases to slow down somewhat.

Upon the second approach $(24 m s \leq \mathrm{t} \leq 35 \mathrm{~ms})$ the convective heat flux increases sharply until the second impact which occurs at $\mathrm{t} \approx 35 \mathrm{~ms}$ where another peak in the convective heat flux is observed. At this time the 
bubble ruptures and the triple contact line spreads causing a short phase of high convective heat flux since Point (b) is outside of the initial rupture region (see Fig. 8). As the triple contact line spreads, Point $(b)$ eventually becomes covered in gas and this causes a rapid drop in the convective heat flux to below the generated heat flux. In fact, upon close inspection of Fig. 11, the convective heat flux becomes slightly negative at $\mathrm{t} \approx 48 \mathrm{~ms}$ and associated with this the storage heat flux becomes larger than the generated heat flux. This suggests that additional heat, over and above that being generated, is being supplied to heat the foil. Since at this moment in time Point (b) is located inside the extremities of the bubble, it cannot be due to warmer liquid underneath the foil. This effect is in fact due to the heated air on the top side of the foil trapped between the foil and the IR glass supplying heat to the foil.

Subsequent to this event the convective heat flux once again rises to a positive value as the foil temperature rises above that of the air in the gap. Here, when the convective heat flux is so low, it is not possible to disentangle the heat flux to the air in the gap from that to the bubble; this is a minor shortcoming of the test facility. Once the bubble is stationary on the surface it assumes a near hemispherical shape, although not shown here.

\subsubsection{Point $c$}

The third point of interest is Point $(c)$. As depicted in Fig. 8, this point is located at the front edge of the bubble during its impact stage and roughly in the centre of the bubble when it is attached to the surface subsequent to the second impact stage.

Referring to Fig. 13 it is seen that during the initial impact there is a spike in convective heat flux which is similar in magnitude to that of Point (a) shown in Fig. 9, although with a delay of approximately $4 \mathrm{~ms}$ i.e $6 \mathrm{~ms}$ after impact. The delay in the initial spike is due to the right hand side (if viewed at an angle of 45 degrees) of the bubble impacting first, followed by the left, such that Point $(c)$ is on the side which impacts last.

Similar to the other two points discussed above the rapid increase in the convective heat flux draws on energy stored within the foil and thus causes the magnitude of the storage heat flux term to increase with an associated decrease in the wall temperature. Between 6 $-14 \mathrm{~ms}$, Fig. 13 shows a decrease in the convective heat flux and, associated with this, a reduction in the rate at which the wall temperature decreases. Interestingly, compared with Point $(a)$ which is also located near the edge of the bubble, the convective heat flux does not drop below zero which indicates that the way in which

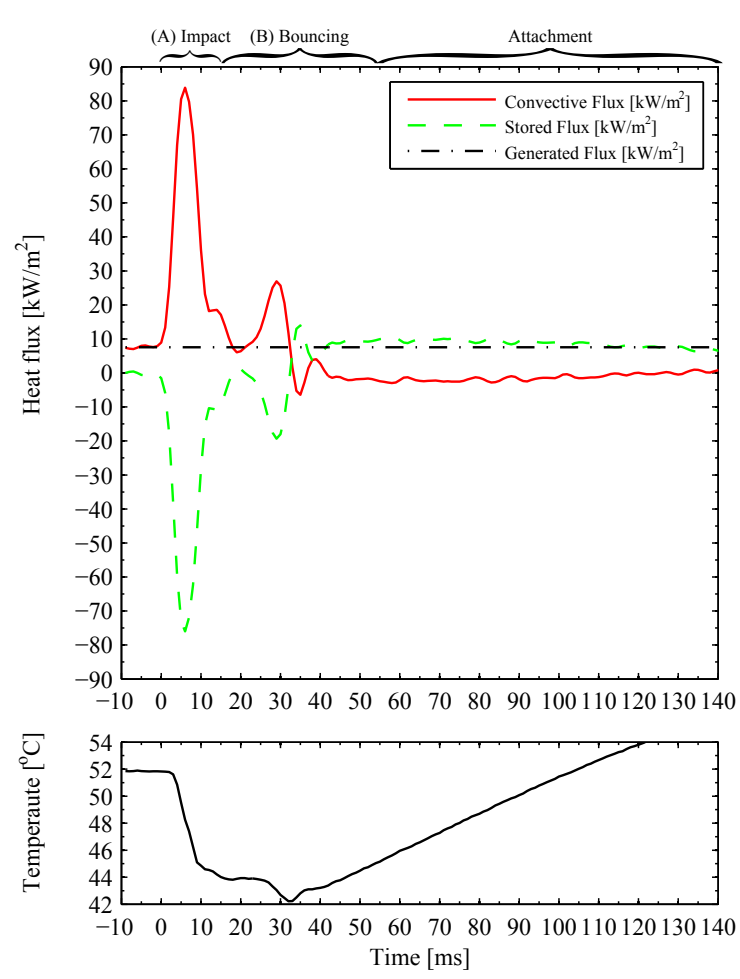

Figure 13: Time trace of heat flux and temperature at Point $c$, shown in Fig. 8. The $x$ axis time begins from when the bubble impacts the surface. The upper graph illustrates the change in surface heat flux, with the lower graph illustrating the change in surface temperature.

the bubble deforms during the impact event makes the heat transfer very specific to the location.

Similar to the trend observed for Point $(b)$, when the bubble begins its rebound away from the surface at $\mathrm{t}$ $=14 \mathrm{~ms}$, there is a notable inflection in the convective heat flux for Point $(c)$ and this is believed to be caused by the bubble recovering its shape as depicted in Fig. 12 (c). As the bubble moves downward away from the surface the convective heat flux decreases to approximately that of the generated heat flux and the wall temperature plateaus.

At around $t=24 m s$ the bubble begins its second approach to the surface resulting in an increased peak in the convective heat flux and a small drop in wall temperature. When the bubble impact, Point $(c)$ is the centre of the impact zone. Since the bubble ruptures immediately, Point $(c)$ is thus exposed to air and the convective heat flux drops below the generated heat flux and the wall temperature begins to increase. A short period of negative convective heat transfer occurs just after the minimum surface temperature has been reached. As dis- 
cussed previously this is due to the warm air above the foil heating the cool surface slightly.

Once the bubble is fully attached to the surface, the surface temperature immediately increases sharply. This temperature increase is a result of the surface being in direct contact with the air within the bubble. The convective heat transfer to the bubble is close to zero whilst the storage heat flux term is comparable to that of the generated heat flux and as a result, the surface temperature increases.

\subsubsection{Point d}
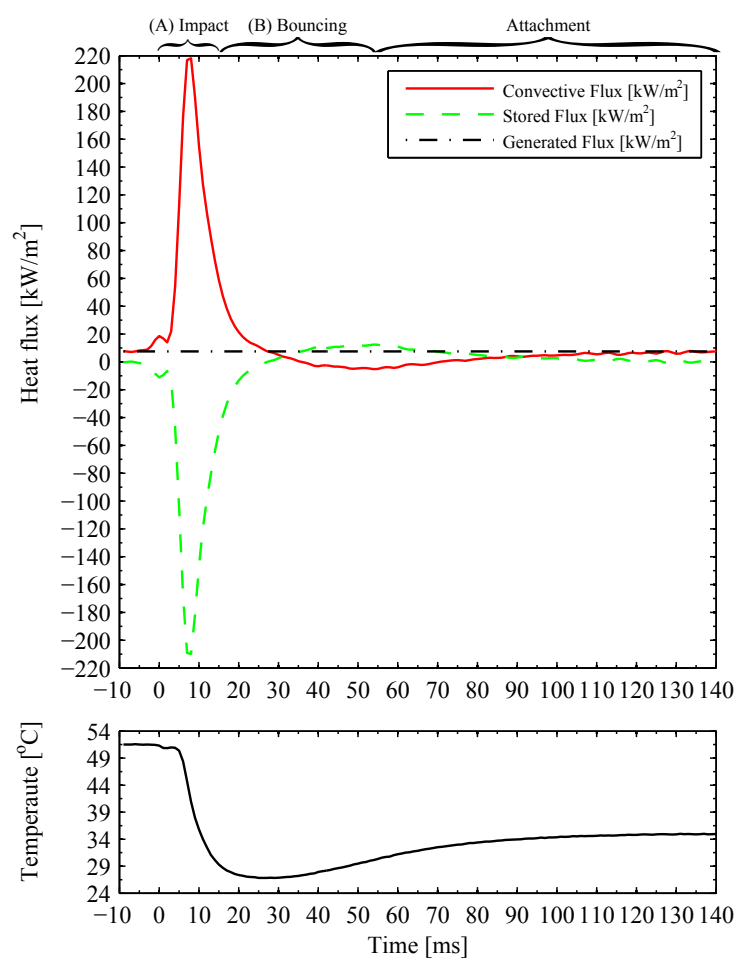

Figure 14: Time trace of heat flux and temperature at Point $d$, shown in Fig. 8. The $x$ axis time begins from when the bubble impacts the surface. The upper graph illustrates the change in surface heat flux, with the lower graph illustrating the change in surface temperature.

The last point of interest is Point $(d)$. As depicted in Fig. 8, this point is located at the first impacting edge of the bubble during its impact stage and is far outside of the bubble when it is attached to the surface subsequent to the second impact stage. As the sequence in Fig. 8 shows, the region where Point $(d)$ is located experiences a markedly different convective heat flux history compared with the other regions.
Fig. 14 illustrates the heat transfer characteristics associated with Point $(d)$ as well as the temperature at that point. At a time of $\mathrm{t}=0 \mathrm{~ms}$, when the bubble has just impacted the surface, there is a small rise in convective flux, which is similar to the trend at Point $(b)$. At $\mathrm{t} \approx 2$ $m s$ the bubble at Point $(d)$ starts to lift from the surface and this is seen in the photographic images of Fig. 8 between $\mathrm{t}=2 \mathrm{~ms}$ and $\mathrm{t}=14 \mathrm{~ms}$. As the bubble lifts it draws in cool fluid and this together with the associated mixing causes the convective heat flux to increase rapidly to a peak of $220 \mathrm{~kW} / \mathrm{m}^{2}$, which is over twice that of the other regions discussed. This being the case, the surface temperature at Point $(d)$ drops notably lower than the others which have been considered. This is an important effect since, when the bubble rebounds off the surface, the heat flux decreases as viscous dissipation settles the fluid motions and the thermal boundary layer re-establishes itself. However, it is noted that the convective heat flux becomes mildly negative for nearly 50 $m s$ subsequent to the second impact. Since Point $(d)$ is located outside of the bubble, the negative heat flux cannot be due to the hot air above the foil, since the heat transfer coefficient to the air layer would be at least an order of magnitude lower than that to the liquid. As a result, this dip in convective heat flux to negative must be due to a negative temperature differential. This is a result of the intense cooling associated with the first impact event causing the temperature to drop to a point that, when fluid is forced over this region during the second impact, it is warmer than the surface causing heat to be added to the foil by convection. The fluid during the second impact is a mixture of hot boundary layer and cool bulk fluid. For $\mathrm{t}>80 \mathrm{~ms}$ the convective heat flux once again becomes positive and approaches that of the generated heat flux. This is to be expected since it is far enough away from the bubble to be uninfluenced by its presence.

\section{Conclusion}

This study presents surface temperature variations and resolved convective heat flux values for a single bubble bouncing upon a $10 \mu \mathrm{m}$ thin foil. The surface temperature has been measured with an IR camera providing $216 \mu \mathrm{m}$ spatial resolution at a frequency of $1 \mathrm{kHz}$. This is used in conjunction with two high speed cameras, which have a spatial resolution of $37 \mu m$, which track the bubble's motion as it leaves an orifice, rises and impacts on the heated surface. Results are presented for a single bubble released from an orifice $30 \mathrm{~mm}$ away from the heated surface, which is initially at a temperature of $51^{\circ} \mathrm{C}$. 
It has been shown that once a bubble departs its growth orifice, it rapidly gains momentum and reaches a maximum terminal velocity of $333 \mathrm{~mm} / \mathrm{s}$ before impacting the solid surface. After the initial impact the bubble was found to rebound from the surface; for the present example only one rebound was observed. The bubble shape was found to vary quite significantly during its rise and even more so during its impact and rebound from the surface.

The effect that a single bouncing bubble has on the surface temperature was found to be significant, with its effect lasting as long as 8 seconds. This lasting effect was found to be related to the wake of the bubble following its initial impact. However the main focus of this study is on heat transfer during the bubble impact and rebound processes. Once the bubble has impacted the surface a substantial variation in convective heat transfer occurred. This variation was found to be spatially symmetric, both within the confines of the bubble and the ensuing wake.

Within an area close to the bubble, substantial and rapid fluctuations in convective heat flux were observed. On some occasions negative heat flux was observed, indicating that warm liquid was momentarily in contact with the previously cooled surface. The fluid dimple formed during the bubble's rise was found to initially retard enhancement, although during the bubble's rebound from the surface, the captured fluid was propelled due to the inversion of the bubble back to a spherical shape. Once the bubble has returned to the surface the next process occurs: rupture of the film separating the bubble from the surface. Once the bubble is fully attached, there is an immediate rise in the surface temperature within the confines of the bubble. Overall the bubble motion is found to enhance convective heat transfer quite significantly.

\section{Acknowledgment}

The authors acknowledge the technical support staff of the Mechanical and Manufacturing Engineering Department of Trinity College Dublin. The project is funded by Science Foundation Ireland (SFI) grant number 09-RFP-ENM2151.

\section{References}

[1] M. A. Atmane, D. B. Murray, The effect of the liquid motion induced by air and vapor bubbles on heat transfer around a cylinder, International Journal of Heat and Mass Transfer 48 (6) (2005) 1084-1095.
[2] K. Cornwell, The influence of bubbly flow on boiling from a tube in a bundle, International Journal of Heat and Mass Transfer 33 (12) (1990) 2579-2584.

[3] A. Sato, M. Shirota, T. Sanada, M. Watanabe, Modeling of bouncing of a single clean bubble on a free surface, Physics of Fluids 23 (2011) 013307.

[4] R. Kirkpatrick, M. Lockett, The influence of approach velocity on bubble coalescence, Chemical Engineering Science 29 (12) (1974) 2363-2373.

[5] H. K. Tsao, D. L. Koch, Observations of high Reynolds number bubbles interacting with a rigid wall, Physics of Fluids 9 (1997) 44.

[6] M. Krzan, K. Lunkenheimer, K. Malysa, Pulsation and bouncing of a bubble prior to rupture and/or foam film formation, Langmuir 19 (17) (2003) 6586-6589.

[7] M. Krasowska, M. Krzan, K. Malysa, Bubble collisions with hydrophobic and hydrophilic surfaces in $\alpha$-terpineol solutions, Physicochemical Problems of Mineral Processing 37 (2003) 3750 .

[8] K. Malysa, M. Krasowska, M. Krzan, Influence of surface active substances on bubble motion and collision with various interfaces, Advances in Colloid and Interface Science 114 (2005) 205-225.

[9] D. Legendre, C. Daniel, P. Guiraud, Experimental study of a drop bouncing on a wall in a liquid, Physics of Fluids 17 (2005) 097105.

[10] J. Zawala, M. Krasowska, T. Dabros, K. Malysa, Influence of bubble kinetic energy on its bouncing during collisions with various interfaces, The Canadian Journal of Chemical Engineering 85 (5) (2007) 669-678.

[11] M. Krasowska, K. Malysa, Kinetics of bubble collision and attachment to hydrophobic solids: I. effect of surface roughness, International Journal of Mineral Processing 81 (4) (2007) 205216.

[12] R. Zenit, D. Legendre, The coefficient of restitution for air bubbles colliding against solid walls in viscous liquids, Physics of Fluids 21 (2009) 083306.

[13] M. Fujasová-Zedníková, L. Vobecká, J. Vejrazka, Effect of solid material and surfactant presence on interactions of bubbles with horizontal solid surface, The Canadian Journal of Chemical Engineering 88 (4) (2010) 473-481.

[14] D. Kosior, J. Zawala, K. Malysa, Influence of n-octanol on the bubble impact velocity, bouncing and the three phase contact formation at hydrophobic solid surfaces, Colloids and Surfaces A: Physicochemical and Engineering Aspects.

[15] D. B. R. Kenning, Y. Yan, Pool boiling heat transfer on a thin plate: features revealed by liquid crystal thermography, International Journal of Heat and Mass Transfer 39 (15) (1996) 31173137.

[16] G. E. Thorncroft, J. F. Klausner, R. Mei, An experimental investigation of bubble growth and detachment in vertical upflow and downflow boiling, International Journal of Heat and Mass Transfer 41 (23) (1998) 3857-3871.

[17] G. E. Thorncroft, J. F. Klausner, The influence of vapor bubble sliding on forced convection boiling heat transfer, Journal of Heat Transfer 121 (1999) 73.

[18] D. Qiu, V. K. Dhir, Experimental study of flow pattern and heat transfer associated with a bubble sliding on downward facing inclined surfaces, Experimental Thermal and Fluid Science 26 (67) (2002) 605-616

[19] B. B. Bayazit, D. K. Hollingsworth, L. C. Witte, Heat transfer enhancement caused by sliding bubbles, Journal of Heat Transfer 125 (3) (2003) 503-510.

[20] Y. M. C. Delauré, V. S. S. Chan, D. B. Murray, A simultaneous PIV and heat transfer study of bubble interaction with free con- 
vection flow, Experimental Thermal and Fluid Science 27 (8) (2003) 911-926.

[21] B. Donnelly, T. S. O'Donovan, D. B. Murray, Bubble enhanced heat transfer from a vertical heated surface, Journal of Enhanced Heat Transfer 15 (2) (2008) 159-169.

[22] B. Donnelly, T. S. O’Donovan, D. B. Murray, Surface heat transfer due to sliding bubble motion, Applied Thermal Engineering 29 (7) (2009) 1319-1326.

[23] D. B. Donoghue, B. Donnelly, D. B. Murray, The enhancement effects of a plume of rising bubbles on natural convection from a heated vertical plate, Journal of Enhanced Heat Transfer 19 (4) (2012) 379-395.

[24] S. Manickam, V. Dhir, Holographic interferometric study of heat transfer to a sliding vapor bubble, International Journal of Heat and Mass Transfer 55 (4) (2012) 925-940.

[25] C. Brücker, Structure and dynamics of the wake of bubbles and its relevance for bubble interaction, Physics of Fluids 11 (1999) 1781-1796.

[26] T. Sanada, M. Shirota, M. Watanabe, Bubble wake visualization by using photochromic dye, Chemical Engineering Science 62 (24) (2007) 7264-7273.

[27] S. Houston, K. Cornwell, Heat transfer to sliding bubbles on a tube under evaporating and non-evaporating conditions, International Journal of Heat and Mass Transfer 39 (1) (1996) 211-214.

[28] Y. Y. Yan, D. B. R. Kenning, I. A. Grant, K. Cornwell, Heat transfer to sliding bubbles under plane and curved surfaces, in: Institution of Mechanical Engineers Conference Publications, Vol. 2, Medical Engineering Publications Ltd, 1995, pp. 295295.

[29] I. Golobic, J. Petkovsek, M. Baselj, A. Papez, D. B. R. Kenning, Experimental determination of transient wall temperature distributions close to growing vapor bubbles, Heat and mass transfer 45 (7) (2009) 857-866.

[30] O. Raghu, J. Philip, Thermal properties of paint coatings on different backings using a scanning photo acoustic technique, Measurement Science and Technology 17 (2006) 2945.

[31] J. Stafford, E. Walsh, V. Egan, Characterizing convective heat transfer using infrared thermography and the heated-thin-foil technique, Measurement Science and Technology 20 (2009) 105401.

[32] I. Golobic, J. Petkovsek, D. B. R. Kenning, Bubble growth and horizontal coalescence in saturated pool boiling on a titanium foil, investigated by high-speed IR thermography, International Journal of Heat and Mass Transfer 55 (4) (2012) 1385-1402.

[33] H. Coleman, W. Steele, Experimentation, validation, and uncertainty analysis for engineers, Wiley, 2009.

[34] J. Kim, T. Simon, R. Viskanta, Journal of heat transfer policy on reporting uncertainties in experimental measurements and results, Journal of Heat Transfer 115 (1993) 5.

[35] Z. Zapryanov, S. Tabakova, Dynamics of bubbles, drops, and rigid particles, Vol. 50, Springer, 1999.

[36] M. H. Hendrix, R. Manica, E. Klaseboer, D. Y. Chan, C.-D. Ohl, Spatiotemporal evolution of thin liquid films during impact of water bubbles on glass on a micrometer to nanometer scale, Physical Review Letters 108 (24) (2012) 247803.

[37] R. Manica, E. Klaseboer, R. Gupta, M. H. W. Hendrix, C.-D. Ohl, D. Y. C. Chan, Modelling film drainage of a bubble hitting and bouncing off a surface, in: Ninth International Conference on CFD in the Minerals and Process Industries, CSIRO, Melbourne, Australia, Vol. 100, 2012, p. 024501. 